at two years in $30 \%$ of cases and progression in $25 \%$; while, at the functional level, $40 \%$ remained stable with worsening in 4 patients per year $(20 \%)$.

$50 \%$ of the patients with SLE received treatment with HCQ and MTX before the diagnosis of ILD; and $35 \%$ corticosteroids $(4+7.3 \mathrm{mg} /$ day). With ILD diagnosed, $\mathrm{HCQ}$ was administered to $95 \%$ of patients and the standard of treatment was based on corticosteroids in monotherapy at a mean dose of $13 \pm 10.5 \mathrm{mg} /$ day due to stability both at radiological level and in function tests respiratory. $25 \%$ and $15 \%$ required treatments such as RTX and BLM as they did not have an adequate response with the previous one.

In our study, we did not find any statistical significance between the variables analyzed, due to the small sample size.

Conclusion: 1)ILD in SLE is a rare manifestation, present in $4.4 \%$ of our series. Standard treatment with hydroxychloroquine and corticosteroids appears to be a useful therapeutic option, stabilizing radiological progression in one third of cases early. More studies with a larger sample size are needed to analyze the role of immunosuppressive treatment in this type of lung involvement.

REFERENCES:

[1] Aguilera-Pickens G, Abud-Mendoza C. Pulmonary Manifestations in Systemic Lupus Erythematosus: Pleural Involvement, Acute Pneumonitis, Chronic Interstitial Lung Disease and Diffuse Alveolar Hemorrhage. Clinical rheumatology. Vol.14 Issue. 5. Pages 294-300 (September-October 2018).

Disclosure of Interests: None declared

DOI: 10.1136/annrheumdis-2021-eular.2700

\section{AB0291 PROGNOSTIC VALUE OF LATE GADOLINIUM ENHANCEMENT ON CARDIAC MAGNETIC RESONANCE IMAGING IN SYSTEMIC LUPUS ERYTHEMATOSUS}

A. J. Velvet ${ }^{1}$, S. Vohra ${ }^{1}$, T. David ${ }^{2}$, B. Keavney ${ }^{3}$, I. Bruce ${ }^{2}$, B. Parker ${ }^{2}$, K. Bratis ${ }^{1}$. ${ }^{1}$ Manchester University NHS Foundation Trust, Cardiology, Manchester, United Kingdom; ${ }^{2}$ Manchester University NHS Foundation Trust, The Kellgren Centre for Rheumatology, Manchester, United Kingdom; ${ }^{3}$ The University of Manchester, NIHR Manchester Musculoskeletal Biomedical Research Centre, Manchester, United Kingdom

Background: Cardiac Magnetic Resonance Imaging (CMRI) with Late Gadolinium Enhancement (LGE) has an established value in the diagnostic and prognostic assessment of ischaemic and non-ischaemic cardiomyopathies. Although CMRI is widely used for the detection of myocardial involvement in subclinical Systemic Lupus Erythematosus (SLE), its prognostic value has not been determined.

Objectives: To determine the prognostic value of CMRI with LGE for major adverse cardiovascular events (MACE) in patients with SLE, and investigate its correlation with the severity of systemic inflammation.

Methods: A retrospective tertiary single-centre review of patients with SLE who underwent a CMRI study at Manchester Foundation Trust between 20092020 was conducted. Patients were categorized into two groups; those who experienced a MACE (cardiac death, myocardial infarction (MI), stroke/TIA or heart failure) and those who did not. We compared cardiovascular (CV) risk factors, CMRI findings, SLE risk scores and biochemistry between the 2 groups.

Results: We identified 20 female patients who underwent a CMRI, with a mean age of 46 years at the time of the scan. Indications for CMRI were assessment for worsening dyspnoea and new onset left ventricular systolic dysfunction. Table 1 demonstrates the clinical, laboratory and CMRI characteristics of the two groups. There were no significant differences in the clinical background and traditional CV risk factors between the 2 groups. 5/20 (25\%) patients experienced a MACE. The SLEDAI-2K score was $>12$ in $2 / 5(40 \%)$ of patients who suffered a MACE and they presented with a stroke within a year of CMRI study, suggesting that systemic inflammation contributes to poor vascular outcomes. $3 / 5(60 \%)$ patients who reported a MACE demonstrated LGE on their CMRI study compared to $3 / 15(20 \%)$ of those who did not (p-0.045). The LGE was predominantly diffuse, mid myocardial in distribution and not ischaemic in pattern, signifying a complex pathophysiological substrate in the development of myocardial pathology in SLE. Additionally, an increase in left ventricular end-diastolic, end-systolic volumes and left atrial diameter was noted in patients who had a MACE $(p<0.05)$. Patients who had a MACE showed a higher incidence of valvular abnormalities and pericardial disease in their CMRI studies. On looking at the medications around the CMRI, the majority of MACE positive patients $4 / 5$ (80\%) were on conventional Disease Modifying Anti-Rheumatic Drugs (DMARDs) such as Mycophenolate, Tacrolimus, Cyclophosphamide, Methotrexate or hydroxychloroquine and none on biologics like Rituximab or Belimumab. While $9 / 15$ of the MACE negative patients were on DMARDs and 4/15 were on biologics.
Table 1. clinical, laboratory and CMRI characteristics of SLE patients with and without MACE. LVEDV-left ventricular end-diastolic volume, LVESV-left ventricular end-systolic volume, LA-left atrial, LVEF-Left ventricular ejection fraction, SLEDAI- SLE Disease Activity Index, +ve-positive.

\begin{tabular}{lcc}
\hline & MACE $+(\mathrm{n}=5)$ & MACE- $(\mathrm{n}=15)$ \\
\hline Mean age of SLE diagnosis (years) & 35.6 & 34.4 \\
Mean duration to CMRI (years) & 8.8 & 11.71 \\
Lupus nephritis & $20 \%$ & $20 \%$ \\
Raynaud's & $40 \%$ & $27 \%$ \\
Previous CV involvement & 0 & 2 \\
SLEDAI-2K > 12 & $2(40 \%)$ & $11(73 \%)$ \\
Lupus anticoagulant +ve & $20 \%$ & $27 \%$ \\
Anticardiolipin +ve & $2(40 \%)$ & $2(14 \%)$ \\
anti-dsDNA (iu/ml)+ve & $3(60 \%)$ & $6(40 \%)$ \\
Low C3 (g/L) & $2(40 \%)$ & $6(40 \%)$ \\
Mean prednisolone dose & 28.33 & 8.21 \\
Conventional DMARDs & $4 / 5(80 \%)$ & $9 / 15(60 \%)$ \\
Biologics & $0 / 5$ & $4 / 15$ \\
Mean LVEF (\%) & 47.4 & 56.7 \\
CMR LGE & $3(60 \%)$ & $3(20 \%)$ \\
Valvular abnormalities & $2(40 \%)$ & $4(27 \%)$ \\
Mean LA area (cm $\left.{ }^{2}\right)$ & 29.6 & 20.6 \\
Pericardial effusion & $40 \%$ & $27 \%$ \\
\hline
\end{tabular}

Conclusion: In our small SLE cohort, we add to existing knowledge that CMRI with LGE is an indispensable tool to investigate cardiac involvement in SLE and may indeed add important prognostic information. Larger scaled studies are required to confirm the use of CMRI with LGE as a predictor of MACE in patients with SLE.

Disclosure of Interests: None declared

DOI: 10.1136/annrheumdis-2021-eular.2748

\section{AB0292 A RETROSPECTIVE STUDY OF PERIOPERATIVE MANAGEMENT OF PATIENTS WITH SLE IN TOTAL HIP ARTHROPLASTY}

I. Kushnareva ${ }^{1}$, M. Makarov' ${ }^{1}$ T. Popkova ${ }^{2}$, A. Khramov ${ }^{1}$, S. Maglevaniy ${ }^{1}{ }^{1}$ V.A. Nasonova Research Institute of Rheumatology, Orthopaedic Department, Moscow, Russian Federation; ${ }^{2}$ V.A. Nasonova Research Institute of Rheumatology, Laboratory of Systemic Rheumatic Diseases, Moscow, Russian Federation

Background: Aseptic necrosis (AN) of bones is one of the most serious complications of systemic lupus erythematosus (SLE), which is characterized by multicomponent joint damage mainly in young people. Long-term observations show that two thirds of patients have multiple aseptic necrosis of bones, with the femoral head being most often affected. Obviously, caused by much strain on the hip joint. In this regard, total hip arthroplasty (THA) is an integral part of the treatment of SLE patients. Despite the fact that THA in these patients allows to achieve good functional outcomes, the amount of complications remains high.

Objectives: To retrospectively analyze the outcomes and structure of complications to determine the tactics of perioperative management of patients with SLE.

Methods: The retrospective group included patients over 18 years of age with a reliable diagnosis of SLE, established according to the classification criteria (SLICC, 2012, ACR, 1997). 123 THA were performed in 77 patients. Outcomes and the structure of complications were analyzed for the period from 1998 to 2016 inclusive.

Results: The period of hospital stay of patients was analyzed. Cementless fixation of the components of the endoprosthesis was used and the friction pair was metal-polyethyleneln all cases. In 23 patients, additional fixation of the acetabular component with screws was performed, which indirectly indicates a poor quality of bone tissue. A more detailed analysis of these patients revealed a long period of glucocorticoid therapy (from 1.5 to 35 years). In 3 patients, during preparation for implantation of the femoral component, a femoral fracture occurred, which required using the cerclages. One patient also had a fracture of the acetabulum, which required the implantation of a Müller anti-protrusio ring. The above-described technical features led to increasing of the total time of surgery, which significantly increased the volume of blood loss. Thus, this required transfusions of blood components: fresh frozen plasma (FFP), erythrocyte suspension, as well as replenishment the circulating blood volume with colloidal solutions.

Prevention of thromboembolism. All patients in the postoperative period underwent common measures of prevention of venous thromboembolism. 